\title{
Atomic ordering of the fluorine dopant in the $\mathrm{HgBa}_{2} \mathrm{CuO}_{4+\delta}$ high- $T_{c}$ superconductor
}

J. G. Correia, ${ }^{* \dagger}$ H. Haas,* and the ISOLDE Collaboration PH Department, CERN, CH-1211 Geneva 23, Switzerland

V. S. Amaral and A. M. L. Lopes

Physics Department, and CICECO, Universidade de Aveiro, 3810-193 Aveiro, Portugal

J. P. Araújo

IFIMUP, Physics Department, FCP, Rua do Campo Alegre 687, P-4150 Porto, Portugal

S. Le Floch

LPMCN, CNRS UMR 5586, Université Lyon-1, Villeurbanne 69622, France

P. Bordet

Laboratoire Cristallographie, CNRS, Avenue des Martyrs 25, F-38042 Grenoble CEDEX 9, France

E. Rita and J. C. Soares

CFNUL, Av. Prof. Gama Pinto 2, P-1699 Lisboa Codex, Portugal

W. Tröger

Fakultät für Physik und Geowissenschaften, Universität Leipzig, Linnéstraße 5, D-04103 Leipzig, Germany

(Received 18 July 2005; revised manuscript received 15 August 2005; published 25 October 2005)

Lattice sites and collective ordering of fluorine atoms in oxygen-reduced samples of $\mathrm{HgBa}_{2} \mathrm{CuO}_{4}$ were investigated with the perturbed angular correlation technique by measuring the electric field gradients induced at ${ }^{199 \mathrm{~m}} \mathrm{Hg}$ nuclei. The experimental data were interpreted with the help of ab initio calculations of charge distributions for different fluorine configurations in $\mathrm{Hg}_{m} \mathrm{Ba}_{2 m} \mathrm{Cu}_{\mathrm{m}} \mathrm{O}_{4 \mathrm{~m}} \mathrm{~F}_{\mathrm{n}}$, supercells. Internal parameters were allowed to relax, to cancel residual atomic forces due to the dopant. The experimental results show clearly that fluorine occupies only the center of the mercury mesh. For a fluorine content $\delta \mathrm{F}>\sim 0.35$ the best agreement with theoretical data is obtained under the assumption that fluorine shows a tendency toward ordering along interstitial rows parallel to $a, b$. In conformity with experimental data from diffraction techniques the calculations show elongations of the $\mathrm{O}(2)-\mathrm{Hg}-\mathrm{O}(2)$ dumbbell and barium shifts towards the fluorine atoms as effects of the dopant. No strong ordering of the transferred charge at the copper planes is present in the computed charge density, even when the fluorine atoms order at high concentrations.

DOI: 10.1103/PhysRevB.72.144523

PACS number(s): 74.72.Jt, 71.15.Mb, 75.40.-s

\section{INTRODUCTION}

After 20 years of intensive research the understanding of the factors leading to high- $T_{c}$ superconductivity in cuprate materials is still far from complete. Being understood that conductivity and magnetism are ruled by the interplay between charge, spin and lattice degrees of freedom, the fact that these phenomena are of short range requires them to be studied at the atomic scale. In fact, numerous and complex structural distortions as polaron deformations, as well as charge and spin stripes, strongly dependent on ionic doping, have been reported in the high- $T_{c}$ copper oxides. In this context, the findings of spin and charge inhomogeneities in the $\mathrm{CuO}$ bonds ${ }^{1}$ and the $\mathrm{CuO}_{2}$ (Ref. 2) layers in $\mathrm{YBa}_{2} \mathrm{Cu}_{3} \mathrm{O}_{6+\delta}$ have been related to lattice deformations. The driving force for the formation of such stripes, alternating regions at the nanometer scale with different structural and electronic properties, may be explained by nonlinear charge ordering and the segregation of holes away from antiferromagnetically ordered regions. Therefore, understanding under which conditions such phenomena occur as well as their possible rela- tionship with chemical structural ordering and if they are connected with high- $T_{c}$ superconductivity is of primary importance. ${ }^{3}$ Recent works propose the concept that the formation of charge inhomogeneity is essential to achieve high$T_{c}$ superconductivity in copper oxides. ${ }^{4}$ It is in this general context that we have studied the simple model compound $\mathrm{HgBa}_{2} \mathrm{CuO}_{4}(\mathrm{Hg} 1201)$ with perturbed angular correlations (PAC) as a local structure technique.

Due to its simple tetragonal structure and highest critical temperatures ${ }^{5}$ the family of the $\mathrm{HgBa}_{2} \mathrm{Ca}_{n-1} \mathrm{Cu}_{\mathrm{n}} \mathrm{O}_{2 \mathrm{n}+2+\delta}$ high$T_{c}$ superconductors is particularly appealing as a system for such investigations. There, $T_{c}$ has been studied as a function of structural lattice constants and internal parameters ${ }^{6}$ and particular attention has been devoted to investigate local distortions, attributed to the excess oxygen $\mathrm{O}_{\delta}$, to understand if these are correlated with the effective doping at the copper planes. ${ }^{7,8}$ Combined x-ray, neutron diffraction, and extended $\mathrm{x}$-ray absorption fine structure (EXAFS) studies performed on $\mathrm{Hg} 1201$ have been claimed to show the existence of distortions at the $\mathrm{Cu}$ planes near $T_{c},{ }^{9,10}$ but without being able to disentangle if these are intrinsic to superconductivity or just 
related with dopant chemical effects. More recently, comparative studies of fluorine $\left(\mathrm{F}_{\delta}^{-}\right)$and oxygen $\left(\mathrm{O}^{2-}{ }_{\delta}\right)$ doping in high quality $\mathrm{Hg} 1201$ samples have been performed. ${ }^{11}$ These works have shown that, on one hand, the effective injection of holes into the $\mathrm{Cu}$ planes is proportional to the dopant nominal charges, as expected from a simple ionic doping model, but, on the other hand, the doping efficiency has remained well below the value expected from the excess fluorine $\mathrm{F}_{\delta}$ concentration in a similar and unexplained way as for $\mathrm{O}_{\delta}$. Hence, the simple idea that the effective hole count in the $\mathrm{Cu}$ planes is just given by the dopant concentrations and ionic charges remains questionable. It is thus of vital importance to know the exact dopant atom concentration at the proper position in the lattice. In spite of a serious effort, doping efficiency studies remain a difficult task with controversial conclusions, mainly attributed to the presence of point defects and impurities. ${ }^{12}$ It is here that PAC can make a decisive contribution by measuring directly the amount of dopant atoms in the lattice, as demonstrated by our work on $\mathrm{O}_{\delta}$ in $\mathrm{Hg} 1201 .^{13}$

As a contribution to understanding the atomistic behavior of the dopant and its influence on local lattice parameters, the present paper describes and discusses the particular case of fluorine doping of oxygen-reduced $\mathrm{Hg} 1201$ high- $T_{c}$ superconductor samples. Fluorine was chosen as the dopant element due to the fact that it has chemistry and ionic radius similar to oxygen, but carries only half the ionic charge. Consequently, for equivalent doping of the charge reservoir, higher atomic concentrations of the dopant atoms are achieved. Upon element doping, the lattice symmetry around the dopant atom is locally modified. One can thus expect that information is obtained about the way that fluorine is accommodated by the mercury planes of $\mathrm{Hg} 1201$ and how the lattice is locally deformed. It might also be possible, in particular, to get a first hint of what happens in $\mathrm{Hg} 1223(n=3)$, the material with the highest $T_{c} \sim 135 \mathrm{~K}$ at ambient pressure, for optimal doping and high excess oxygen concentration of $\delta \mathrm{O} \sim 0.44$.

In the present work the dopant behavior is probed at the same lattice scale that local phenomena actually happen. The major result is the experimental finding that for excess fluorine content $\delta \mathrm{F}>\sim 0.35$, interstitial fluorine orders with short coherence length along rows parallel to the $a$ (or $b$ ) axis in the mercury planes, already in the normal state. On the basis of charge density obtained in our first-principles calculations, performed to interpret the PAC data, it is further proposed that the lattice deformations induced by the dopant and local dopant ordering do not interfere with possible charge ordering at the $\mathrm{Cu}$ planes.

On a more general basis, the experimental methods used here can also be expected to be particularly useful when studying other oxides where the lattice structure and electric properties depend on local phenomena, with still unclear correlations. ${ }^{14}$

\section{EXPERIMENT}

$\mathrm{HgBa}_{2} \mathrm{CuO}_{4+\delta}(\mathrm{Hg} 1201)$ overdoped powder was synthesized at CNRS Grenoble according to Ref. 15. The powder

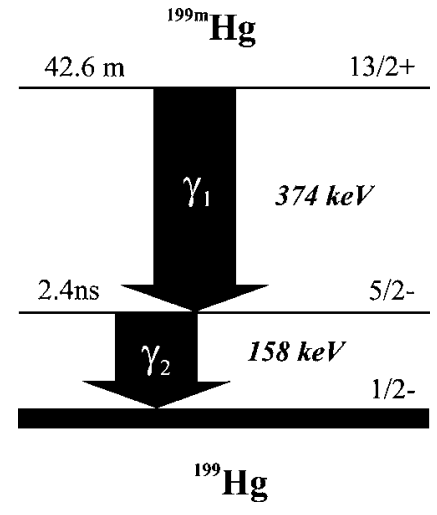

FIG. 1. $\gamma$ - $\gamma$ decay cascade of the ${ }^{199 \mathrm{~m}} \mathrm{Hg}\left(T_{1 / 2}=42.6 \mathrm{~min}\right)$ isomeric state.

was then placed in a vacuum-sealed silica tube together with a zirconium foil, used as an oxygen getter. After annealing for $50 \mathrm{~h}$ at $350{ }^{\circ} \mathrm{C}$ the powder is a single $\mathrm{Hg} 1201$ underdoped phase with $T_{c} \sim 41 \mathrm{~K}$. Roughly $0.2 \mathrm{~g}$ of this material was sent under nitrogen atmosphere to ISOLDE/CERN. ${ }^{16}$ The PAC experiments were performed in a similar way as reported in Ref. 13, where $\mathrm{Hg} 1201$ has been studied as a function of $\mathrm{O}_{\delta}$ concentration. All handling of the material was done in a glove-box filled with dry nitrogen, equipped with a nitrogen ventilated feed trough and a small tubular quartz furnace for annealing under argon atmosphere. Each PAC sample consisted of $\sim 0.2-0.3 \mathrm{mg}$ of powder, homogeneously implanted at $60 \mathrm{keV}$ with radioactive ${ }^{199 \mathrm{~m}} \mathrm{Hg}\left(T_{1 / 2}\right.$ $=42 \mathrm{~min}$ ) to a low dose of $3.5 \times 10^{12}$ at. $\mathrm{cm}^{-2}$. The mean implantation depth was calculated to be about $17 \mathrm{~nm}$ in the powder grains. ${ }^{17}$ The implantations and sample transfer between the glove box and the implantation chamber were done in vacuum. After implantation each sample was first annealed for $18 \mathrm{~min}$ at $190-200{ }^{\circ} \mathrm{C}$ under argon flow to remove remaining implantation defects. ${ }^{13}$ Following this step the powder was enclosed in highly purified vacuum baked copper tubes. These were then sealed with a special highvacuum-purpose pressure-sealing tool. For doping with fluorine, a small grain of $\mathrm{XeF}_{2}$ of about $0.1-0.3 \mathrm{~mm}^{3}$ was enclosed together with the sample. The concentration in the probing region could be estimated by the analysis of the PAC data. The sealed copper sample holders were then removed from the glove box and inserted into a precision temperature controlled furnace at the center of a highly efficient six $\gamma$-ray detector PAC setup as described in Refs. 13 and 18.

The relevant information, i.e., the electric field gradient (EFG) induced by the charge distribution at the mercury nuclei, modulates the half-life histogram of the $2.4 \mathrm{~ns}, 5 / 2,158$ $\mathrm{keV}$ intermediate state of the ${ }^{199 \mathrm{~m}} \mathrm{Hg}$ cascade (Fig. 1). Thirty $\gamma_{1}(374 \mathrm{keV})-\gamma_{2}(158 \mathrm{keV}) \quad$ coincidence time spectra, $N_{j}(\theta, t)$, from detector pairs with relative angles $\theta=180^{\circ}(j$ $=6)$ and $\theta=90^{\circ}(j=24)$ are recorded. After chance coincidences subtraction the systematic efficiency errors and the half-life exponential component are eliminated by constructing the experimental ratio function $R(t)$ according Eq. (1) to reveal the perturbation function, as described in more detail in Ref. 18. 


$$
R(t)=\frac{\sqrt[6]{\prod_{j}^{6} N_{j}\left(180^{\circ}, t\right)}-\sqrt[24]{\prod_{i}^{24} N_{i}\left(90^{\circ}, t\right)}}{\sqrt[6]{\prod_{j}^{6} N_{j}\left(180^{\circ}, t\right)}+2 \sqrt[24]{\prod_{i}^{24} N_{i}\left(90^{\circ}, t\right)}} .
$$

For each angle $\theta$, angular correlation functions $W(\theta, t)$ are calculated numerically by taking into account the full Hamiltonian for the nuclear quadrupole hyperfine interaction. ${ }^{19}$ This function is essentially a product of the angular correlation coefficient, solely determined by the nuclear decay characteristics, and a time-dependent perturbation function describing the oscillation of the nuclear spin system. For a purely magnetic interaction this would be a simple rotation, the Larmor precession. For our case of pure nuclear quadrupole interaction there are several oscillating frequencies contributing. Equation (2) defines the theoretical function, $R_{\mathrm{fit}}(t)$, whose parameters are fitted to the experimental $R(t)$ function

$$
R_{\mathrm{fit}}(t)=2 \frac{W\left(180^{\circ}, t\right)-W\left(90^{\circ}, t\right)}{W\left(180^{\circ}, t\right)+2 W\left(90^{\circ}, t\right)} .
$$

For a $\gamma-\gamma$ cascade with the intermediate level of spin $I$ $=5 / 2$, three frequencies are observable per EFG. From these frequencies the coupling constant of the interaction $\nu_{Q}$ $=e Q V_{z z} / h$ and the asymmetry parameter $\eta=\left(V_{x x}-V_{y y}\right) / V_{z z}$ are extracted. $V_{z z}$ is the principal component of the EFG tensor that is produced by the charge distribution in the surroundings of the probing nucleus. $V_{y y}$ and $V_{x x}$ are the components of the tensor along the $y$ and $x$ axes, which are chosen according $\left|V_{z z}\right|>\left|V_{y y}\right|>\left|V_{x x}\right|$. In the case of an interaction with randomly distributed defects a distribution of frequencies is observed, which broadens the frequency spectrum and attenuates the $R(t)$ as a function of time. In this work only weak distributions around a central $\nu_{Q}$ value were observed, which were assumed to be Lorentzian-like. Their width is characterized by $\sigma_{Q}=$ full width at half maximum $(\mathrm{FWHM}) / 2$ that depends on the density and variety of the lattice defects. ${ }^{20}$

By measuring the electric field gradients, EFGs, at ${ }^{199 m} \mathrm{Hg}$ nuclei, the mercury neighborhood is probed as a function of $\delta \mathrm{F}$. The results are interpreted with the help of $a b$ initio charge density calculations in relaxed superlattices for different configurations of the dopant $\mathrm{F}_{\delta}$.

\section{RESULTS}

\section{A. Experiments}

Figure 2 (left) shows the $R(t)$ functions and (right) the corresponding Fourier transforms (FT) as measured under different experimental conditions. The high-quality fits (reduced $\chi^{2}$ close to 1) are shown by continuous lines in the $R(t)$ spectra and are included as thicker lines on the FT plots. The resulting fit parameters are summarized in Table I. When performing the first annealing step at $200{ }^{\circ} \mathrm{C}$ under argon flow, the spectrum of Fig. 1(a) shows a frequency triplet that reveals only one axially symmetric $\mathrm{EFG}_{1}$, characterized by $v_{Q 1}=1440 \pm 15 \mathrm{MHz}$ and $\eta_{1}=0$. This reproduces the value

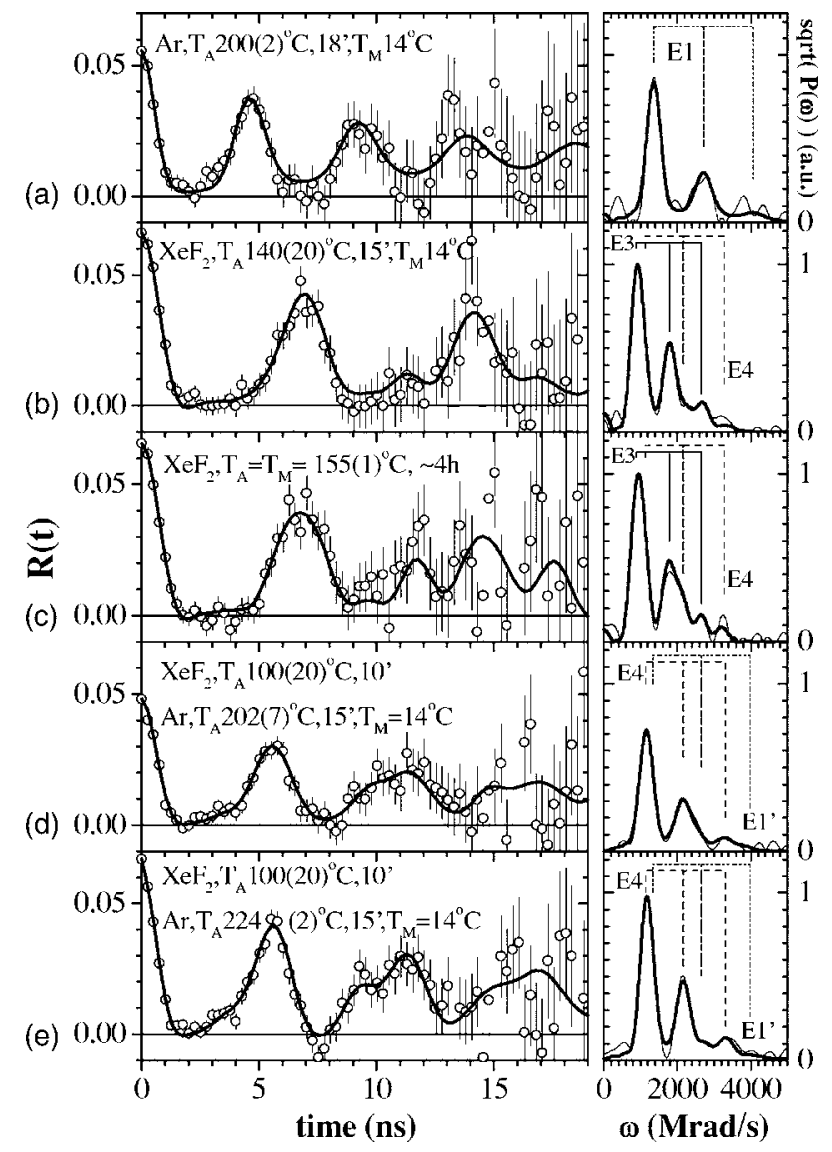

FIG. 2. (a)-(e) (left) experimental $R(t)$ and fit functions and (right) their corresponding Fourier transforms. Each EFG (abbreviated by $E$ ) is represented by its respective frequency triplet, plotted as vertical lines.

found in Ref. 13 for ${ }^{199 \mathrm{~m}} \mathrm{Hg}$ nuclei at regular sites of the $\mathrm{Hg} 1201$ lattice with local tetragonal symmetry, without $\mathrm{O}_{\delta}$ or other point defects in their neighborhood. An attenuation of the $R(t)$ is still observable with $\sigma_{\mathrm{Q} 1}=102 \pm 15 \mathrm{MHz}$. Most likely, few remaining and uncorrelated mercury vacancies produce this EFG distribution. Figures 1(b) and 1(c) clearly show how the spectra change when performing a second annealing step under $\mathrm{XeF}_{2}$. Now, a main fraction $f_{3}$ $=68 \%-80 \%$ of the mercury atoms shows a new $\mathrm{EFG}_{3}$, characterized by mean values $v_{Q 3}=941 \pm 6 \mathrm{MHz}$ and $\eta_{3}$ $=0.019 \pm 0.004$. Smaller fractions of mercury atoms interact with an asymmetric $\mathrm{EFG}_{4}$, with mean values $v_{Q 4}$ $=1138 \pm 13 \mathrm{MHz}$ and $\eta_{4}=0.20 \pm 0.09$. For samples where, after annealing under $\mathrm{XeF}_{2}$, a third annealing step was done under argon, the spectra change once more as shown in Figs. 1(d) and 1(e). Mainly one field is observable that affects $f_{4}$ $=70 \%-87 \%$ of the mercury atoms. Being characterized by mean value $v_{Q 4}=1159 \pm 6 \mathrm{MHz}$ and a nonzero asymmetry parameter $\eta_{4}=0.215 \pm 0.007$ it is assigned to be the same as $\mathrm{EFG}_{4}$ above. In these samples, small fractions of mercury atoms are again found interacting with a new $\mathrm{EFG}_{1}{ }^{\prime}$ with nil asymmetry parameter and, when compared to $\mathrm{EFG}_{1}$, with slightly lower $v_{Q}$ values. $\mathrm{EFG}_{1}{ }^{\prime}$ evidences mercury atoms without defects or fluorine in their neighborhood. It is assigned in the Fourier spectra of Figs. 1(d) and 1(e) and pa- 
TABLE I. EFG fitting parameters of the experimental $R(t)$ PAC functions, together with a shorthand description of the sample preparation.

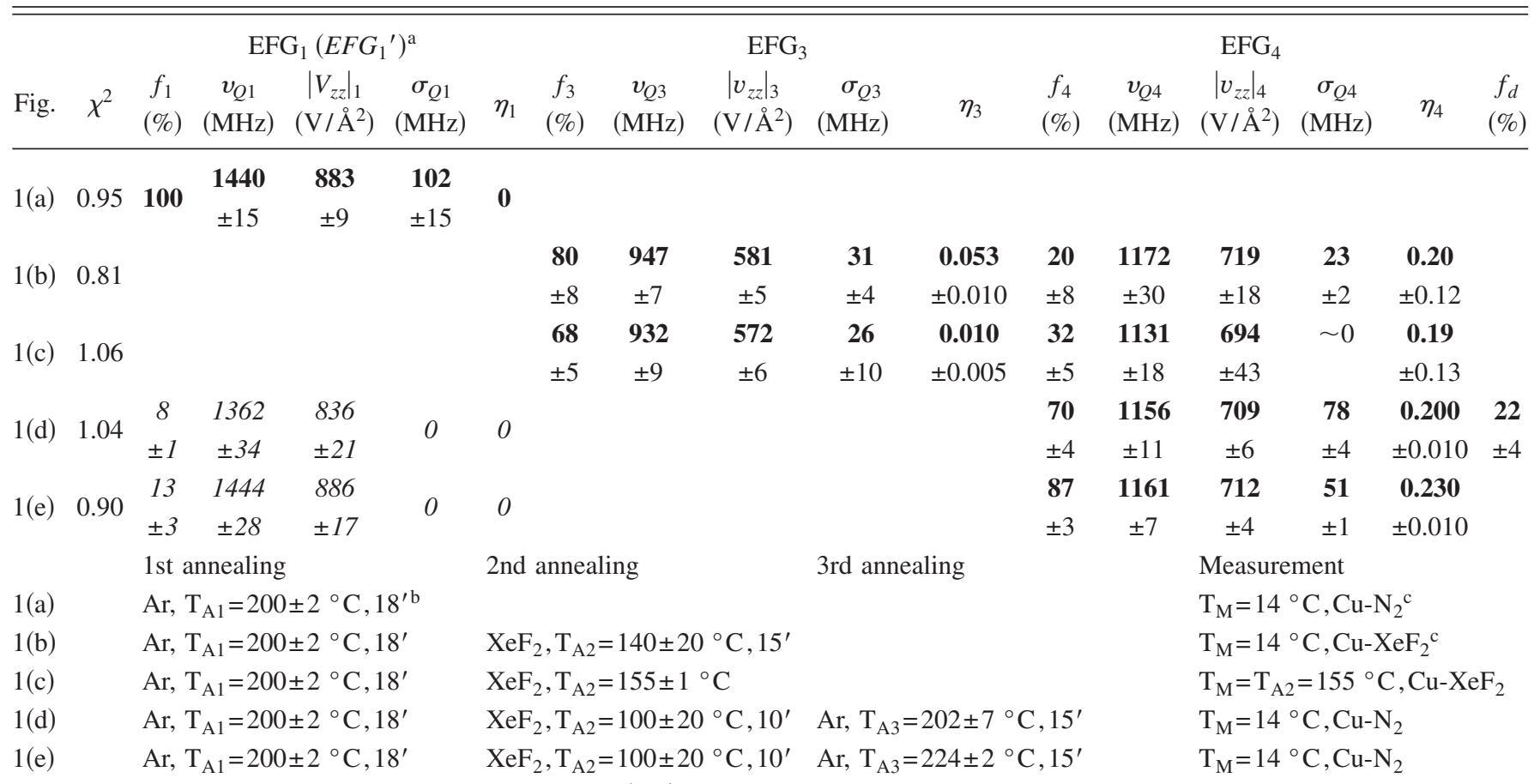

Notes: The measurement time per sample is $\sim 4 \mathrm{~h}$. The $\left|V_{z z}\right|$ quoted errors are relative fit errors. The absolute error is given by $\delta Q / Q$ $\sim 11.5 \%$.

atalic values denote fields assigned in Fig. 1 as $\mathrm{EFG}_{1}{ }^{\prime}$ for (d) and (e); see text.

${ }^{\mathrm{b}} \mathrm{T}_{\mathrm{A}}=$ Annealing temperature, $\mathrm{T}_{\mathrm{M}}=\mathrm{PAC}$ measurement temperature.

" $\mathrm{Cu}-\mathrm{N}_{2}$ " or " $\mathrm{Cu}-\mathrm{XeF}_{2}$ " denote PAC measurements performed under $\mathrm{N}_{2}$ or under $\mathrm{XeF}_{2}$ in sealed $\mathrm{Cu}$ tube.

rameterized by italic values in Table I. The lower $v_{Q}$ value could possibly be explained by an extra elongation of the average mercury-apical oxygen bond distance, $d[\mathrm{O}(2)-\mathrm{Hg}]$, upon fluorine doping, as observed in neutron diffraction data. ${ }^{11}$ In Fig. 1(d) after the third annealing step under argon, the amplitude of the spectrum is slightly reduced. This is attributed to an extra fraction $f_{\mathrm{d}}=22 \%$ of mercury nuclei, which are interacting with a very wide $\mathrm{EFG}_{\mathrm{d}}$ distribution of unknown origin, that cannot be resolved experimentally.

\section{B. Density functional calculations}

Semirelativistic electronic structure calculations were performed with the $a b$ initio full potential linearized augmented plane wave (FLAPW) code WIEN (Ref. 21) using the generalized gradient approximation ${ }^{22}$ (GGA) for the density functional. This method allows, in particular, the computation of hyperfine parameters in crystalline solids by only requiring the lattice constants as input parameters. Technical details were similar to our earlier work. ${ }^{13}$ To account for the fluorine $\mathrm{F}_{\delta}$ doping, several supercells were constructed with composition $\mathrm{Hg}_{\mathrm{m}} \mathrm{Ba}_{2 \mathrm{~m}} \mathrm{Cu}_{\mathrm{m}} \mathrm{O}_{4 \mathrm{~m}} \mathrm{~F}_{\mathrm{n}}$, assuming $\delta \mathrm{O}=0$. Only configurations which provide EFGs relevant for the explanation of the experimental data are reported here. These have $\mathrm{F}_{\delta}$ atoms occupying exclusively $[ \pm 1 / 2, \pm 1 / 2,0]$ sites in the mercury planes, but with different arrangements depending on their concentration.
Figure 3 shows atomic projections of these superlattices onto the mercury planes, as viewed along the crystalline $c$ axis, where shaded regions represent the $a b$ plane of the supercells used in the FLAPW simulations. The undoped D1 supercell has $m=1$ and $n=0$ (not shown). The $D 4$ supercell, $m=4, n=1$, corresponding to $\delta \mathrm{F}=0.25$, has mercury atoms interacting with a single $\mathrm{F}_{\delta}$ neighbor. Two different local configurations $D 2 a$ and $D 2 b$ exist for mercury interacting with two $\mathrm{F}_{\delta}$, both represented by $m=2, n=1$ supercells, with corresponding $\delta \mathrm{F}=0.50$. $D 2 a$ distributes $\mathrm{F}_{\delta}$ as on a chessboard pattern, where two fluorine atoms are aligned with one mercury atom. In $D 2 b$, fluorine is distributed along interstitial rows parallel to the $b$ axis. The two fluorine-mercury bonds in this case make approximately a $90^{\circ}$ angle. The data analysis has motivated further simulations of a D3 supercell, $m=3, n=1$, with more diluted fluorine concentrations, $\delta \mathrm{F}$ $=0.33$, where the fluorine atoms are arranged as in $D 2 b$. For all supercells the internal parameters were allowed to relax to positions with vanishing internal forces. The lattice parameters $a$ and $c$ were fixed to the values for pure $\mathrm{Hg} 1201$ to keep the computer effort within the limits of the available resources.

Table II lists the EFG parameters found for each atom, as calculated from the corresponding charge distribution, in each relaxed supercell. Table III shows the corresponding geometrical parameters. Figure 4 shows a three-dimensional (3D) artistic view of the relaxed $D 4, D 3, D 2 a$, and $D 2 b$ supercells. The atomic internal parameter shifts were calculated 


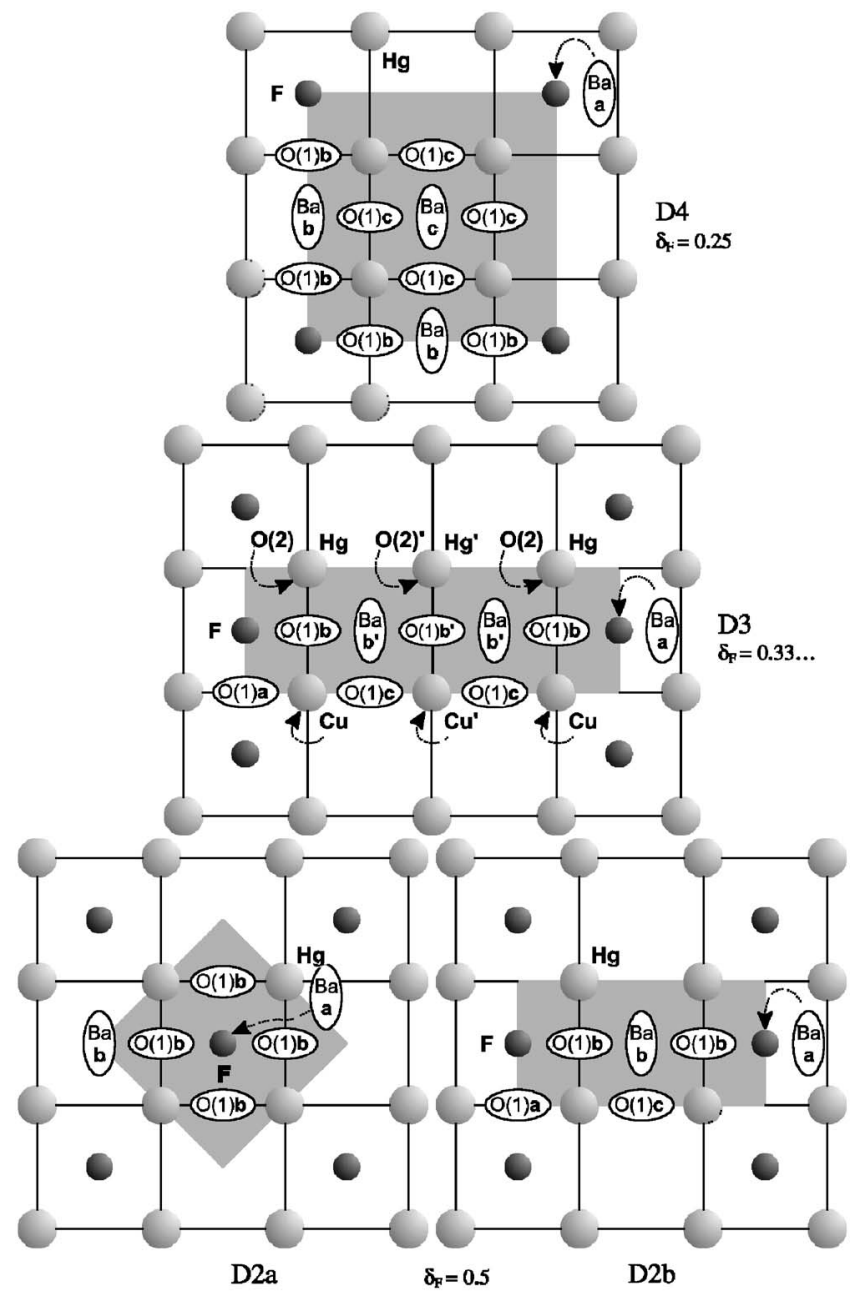

FIG. 3. Representation of the $\mathrm{Hg}$ planes of $\mathrm{Hg} 1201$ as viewed along the $c$ axis. Shaded regions show the different supercells used into the FLAPW simulations. Symbols enclosed into ellipses assign the projected atomic positions of nonequivalent $\mathrm{Ba} \_a, \mathrm{Ba} \_b, \mathrm{Ba} \_b^{\prime}, \mathrm{Ba} \_c$ atoms and of the copper plane oxygen, $\mathrm{O}(1) \_a, \mathrm{O}(1) \_b, \mathrm{O}(1) \_b^{\prime}, \mathrm{O}(1) \_c$ atoms. Copper and the apical oxygen $\mathrm{O}(2)$ atoms are shadowed by $\mathrm{Hg}$ atoms. Hence, black arrows point to their projected location. In the case of the D3 configuration, there are nonequivalent $\mathrm{Hg}, \mathrm{O}(2)$ and $\mathrm{Cu}$ sites which are indicated by a prime notation. Relaxation effects are not shown in this figure.

relative to the undoped $D 1$ supercell and are amplified by a factor of 10 , to make the atomic displacements visually observable.

\section{DISCUSSION}

We start by interpreting the results of our theoretical calculations, since they will serve later in the analysis of the experimental data. The theoretically obtained geometry changes on fluorine doping shall be discussed first. Unfortunately at the present state of the calculations we are not able to compute the changes of the absolute lattice constants, quite well known from experiment. We thus have to limit the comparison with experiment to the internal structural param- eters, rather imprecisely known from the few neutron diffraction measurements. Figure 5 shows the calculated individual internal position parameters $\mathrm{z}$ for the apical oxygen, $\mathrm{O}(2)$, and the Ba sites as a function of $\delta \mathrm{F}$, plotted together with the measured average parameters. ${ }^{11}$ The experimental values for the undoped, $\delta F=\delta O=0$, case were obtained by extrapolation of more recent results. ${ }^{23}$ The calculations reproduce the measured trends, though no fully quantitative agreement is obtained. Contrary to the results quoted in Ref. 11 we find theoretically a stronger shift in the $\mathrm{O}(2)$ position for $\mathrm{O}_{\delta}$ than for $\mathrm{F}_{\delta}$ doping by a factor of 2 , as might be expected from a simple charge doping model. The present calculations also result in strongly different $z$ coordinates for the nonequivalent Ba sites in each supercell, in particular. In Fig. 5 they are plotted together with their multiplicity weighted average to compare with the experimental values. Ba atoms above/ below $\mathrm{F}_{\delta}$ show the strongest shift towards the mercury plane, as could be expected from the electrostatic attraction by negatively charged F. In general it can be stated, that the theoretically obtained geometry changes are in quite good agreement with the scarce experimental information available from neutron diffraction.

The calculated EFG parameters, $V_{z z}$ and $\eta$, at the mercury site with increasing $\delta \mathrm{F}$ are the primary theoretical results for comparison with our experiments. The magnitude $\left|V_{z z}\right|$ decreases with doping, as also observed for oxygen in our earlier work. ${ }^{13}$ The analysis of the theoretical results shows that this effect may be attributed to the electrostatic interaction with the fluorine atoms and the $d[\mathrm{Hg}-\mathrm{O}(2)]$ elongation, both of about equal importance. The undoped $D 1$ case has the highest $\left|V_{z z}\right|$ with $\eta_{D 1}=0$ due to local tetragonal symmetry. The $D 4$ configuration shows a nonsymmetric EFG, $\eta_{D 4}$ $\sim 0.2$, with a lower $\left|V_{z z}\right|_{D 4}$ value. Both configurations $D 2 a$ and $D 2 b$ have similar and even lower $\left|V_{z z}\right|_{D 2}$. In $D 2 a$ the two $\mathrm{Hg}-\mathrm{F}$ bonds make a $180^{\circ}$ angle in the $a b$ plane and the resulting EFG component leads to a sizeable asymmetry parameter $\eta_{D 2 a}=0.42$, close to twice the value calculated for $D 4$. In $D 2 b$, on the other hand, the corresponding angle is $90^{\circ}$ and the EFG components along $x$ and $y$ become almost equal, producing $\eta_{D 2 b} \sim 0$. The consistency of the simulations and their interpretation is reinforced by the results obtained with the supercell $D 3$, with an intermediate $(\delta \mathrm{F}$ $=0.33 \ldots)$ fluorine concentration. In these simulations several aspects are revealed which stress in particular the local influence of the fluorine atom on the EFG at the probing mercury atoms. For $\mathrm{Hg}^{\prime}{ }_{D 3}$ nuclei, which have no fluorine in the immediate neighborhood, the simulated $V_{z z}\left(\mathrm{Hg}^{\prime}\right)_{D 3}$ $=-741.1 \mathrm{~V} / \AA^{2}$ and almost nil $\eta\left(\mathrm{Hg}^{\prime}\right)_{D 3}=0.023$ are extremely close to the ones found for mercury in the undoped $D 1$ configuration. This means that in the D3 configuration, $\mathrm{Hg}^{\prime}$ nuclei without $\mathrm{F}$ atoms nearby behave as in an essentially free environment. In accordance with this interpretation is the fact that the $d\left[\mathrm{Hg}^{\prime}-\mathrm{O}(2)\right]$ distance is practically unchanged. In addition, the values found for $V_{z z}(\mathrm{Hg})_{D 3}$ $=-640.7 \mathrm{~V} / \AA^{2}$ and almost nil $\eta(\mathrm{Hg})_{D 3}=0.013$ are extremely close to the values found for the $V_{z z}(\mathrm{Hg})_{D 2 b}$ configuration, again in agreement with a local picture for the EFG at $\mathrm{Hg}$.

A completely opposite picture for the charge distribution in the $\mathrm{Cu}$ planes is obtained from our calculations, however. 
TABLE II. Calculated EFG parameters for all atoms on the relaxed $\mathrm{Hg} 1201+\mathrm{F}$ superlattices. The inserted figures show the Hg planes of different supercells, presented as shadowed regions. Light/dark symbols represent $\mathrm{Hg} / \mathrm{F}$ atoms.

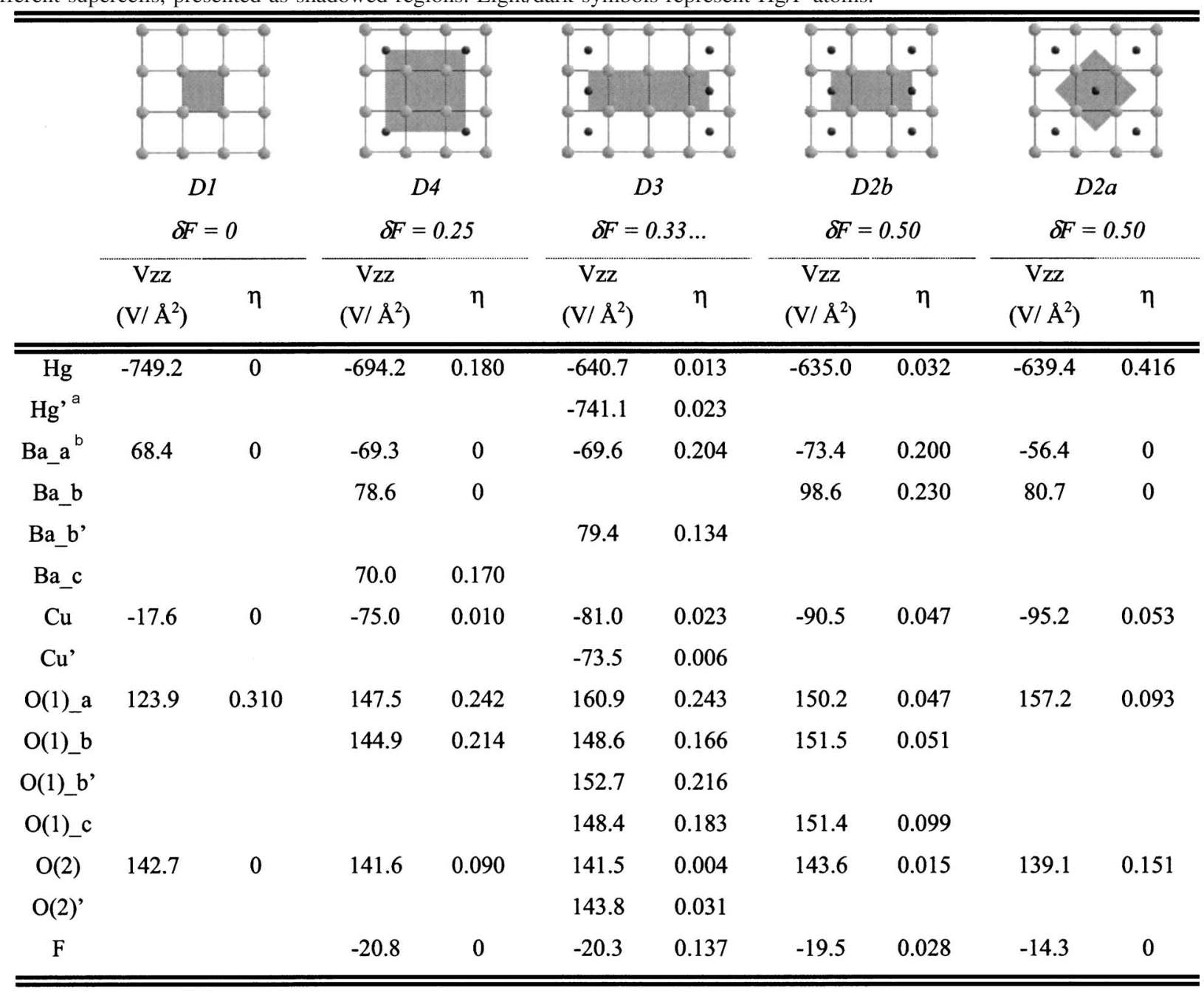

a prime quoted notations refer to non-equivalent sites, which are only observable in the $D 3$ supercell configuration.

' $\mathrm{X} \_\mathrm{a}, \mathrm{X} \_\mathrm{b}$ or X_c, denote non-equivalent $\mathrm{X}$ element sites.

The D3 configuration is the only one calculated here with nonequivalent $\mathrm{Hg}$ and $\mathrm{Cu}$ atoms, regarding the fluorine distribution. In contrast to the $\mathrm{Hg}$ case discussed above, the $V_{z z}$ values for the nonequivalent $\mathrm{Cu}$ and $\mathrm{Cu}^{\prime}$ atoms in this supercell are quite similar, and deviate strongly from the undoped case. In spite of the fact that the calculated EFGs at copper atoms in high- $T_{c}$ compounds are not reproducing the experimental values well, we can treat this as evidence for a delocalized charge distribution in the $\mathrm{Cu}$ planes. Also an analysis of the effective oxidation state as estimated from the filling of the $\mathrm{Cu} d$ bands shows no significant difference in the charge transfer for the two sites. Therefore the charge distribution in the copper planes appears to be rather spread out and not much influenced by local structuring of the fluorine dopant. The EFG values calculated at the $\mathrm{Ba}$ sites are extremely sensitive on the local geometry, even changing sign for the $\mathrm{Ba}$ atoms below/above the dopant $\mathrm{F}$.

Our experimental conclusions to be discussed below have prompted us to look at the calculated enthalpy difference between the $D 2 b$ and $D 2 a$ configurations. The total energy difference found of $0.12 \mathrm{eV}$ is larger than the estimated accuracy. Thus the present simulations seem to indicate a small preference of $D 2 b$ relative to $D 2 a$. Preliminary calculations for the $D 2 b$ supercell were then performed with $a$ and $b$ left free to vary at constant cell volume. The energy difference relative to $D 2 a$ was found to be further increased by about $0.02 \mathrm{eV}$ with a $1 \%$ elongation of the $b$ parameter along the fluorine rows. As the present calculation methods are very similar to the ones used in Ref. 24 for $\mathrm{Hg} 1212$, we as well observed that only a fraction of the doping charge is transported to the $\mathrm{Cu}$ planes, with no relevant differences regarding $D 2 a$ and $D 2 b$.

We now turn to the interpretation of the results from our PAC measurements. From chemical and diffraction studies it is well known that the mercury planes in $\mathrm{Hg} 1201$ can have a very high concentration of fluorine following annealing under $\mathrm{XeF}_{2}$. This is also consistent with the know fact that this material can easily load much more $\mathrm{F} \delta$ than $\mathrm{O} \delta$, due to the 
TABLE III. Internal cell parameters calculated for the relaxed $\mathrm{Hg} 1201+\mathrm{F}$ superlattices. Inserted figures as in Table II.

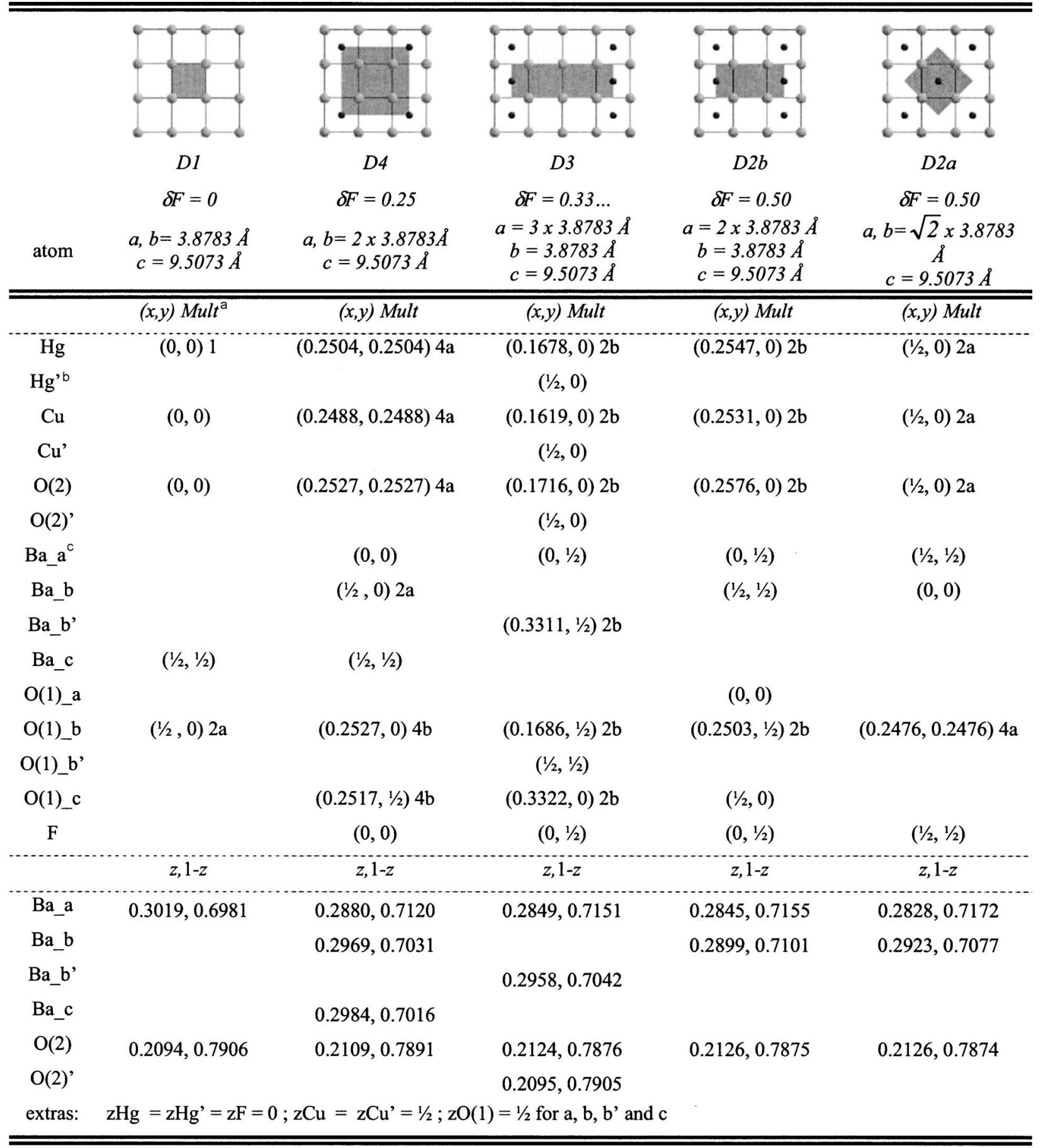

${ }^{a}$ Multiplicities: $2 \mathrm{a} \rightarrow(\mathrm{x}, \mathrm{y}),(\mathrm{y}, \mathrm{x}) ; 2 \mathrm{~b} \rightarrow(\mathrm{x}, \mathrm{y}),(-\mathrm{x}, \mathrm{y}) ; 4 \mathrm{a} \rightarrow(\mathrm{x}, \mathrm{y})(\mathrm{x},-\mathrm{y})(-\mathrm{x}, \mathrm{y})(-\mathrm{x},-\mathrm{y}) ; 4 \mathrm{~b} \rightarrow(\mathrm{x}, \mathrm{y})(-\mathrm{x}, \mathrm{y})(\mathrm{y}, \mathrm{x})(\mathrm{y},-\mathrm{x})$

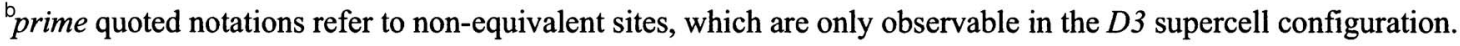

X_a, X_b or X_c, denote non-equivalent X element sites.

halved charge doping efficiency. Following the PAC experiments, x-ray analysis of our samples has shown the Hg1201 phase to be present as the only or strongly dominant component. Because of the small sample size, however, no complementary quantitative information on $\delta_{\mathrm{F}}$ could be obtained from these measurements.
From the preparation conditions and chemical arguments we can infer that sample 1a should be only slightly doped pure $\mathrm{Hg} 1201$, while samples $1 \mathrm{~b}$ and 1c should have the highest fluorine content. Samples 1d and 1e are expected to have an intermediate F concentration. Qualitative inspection of our PAC spectra confirms the expected grouping. Samples $1 \mathrm{~b}$ 


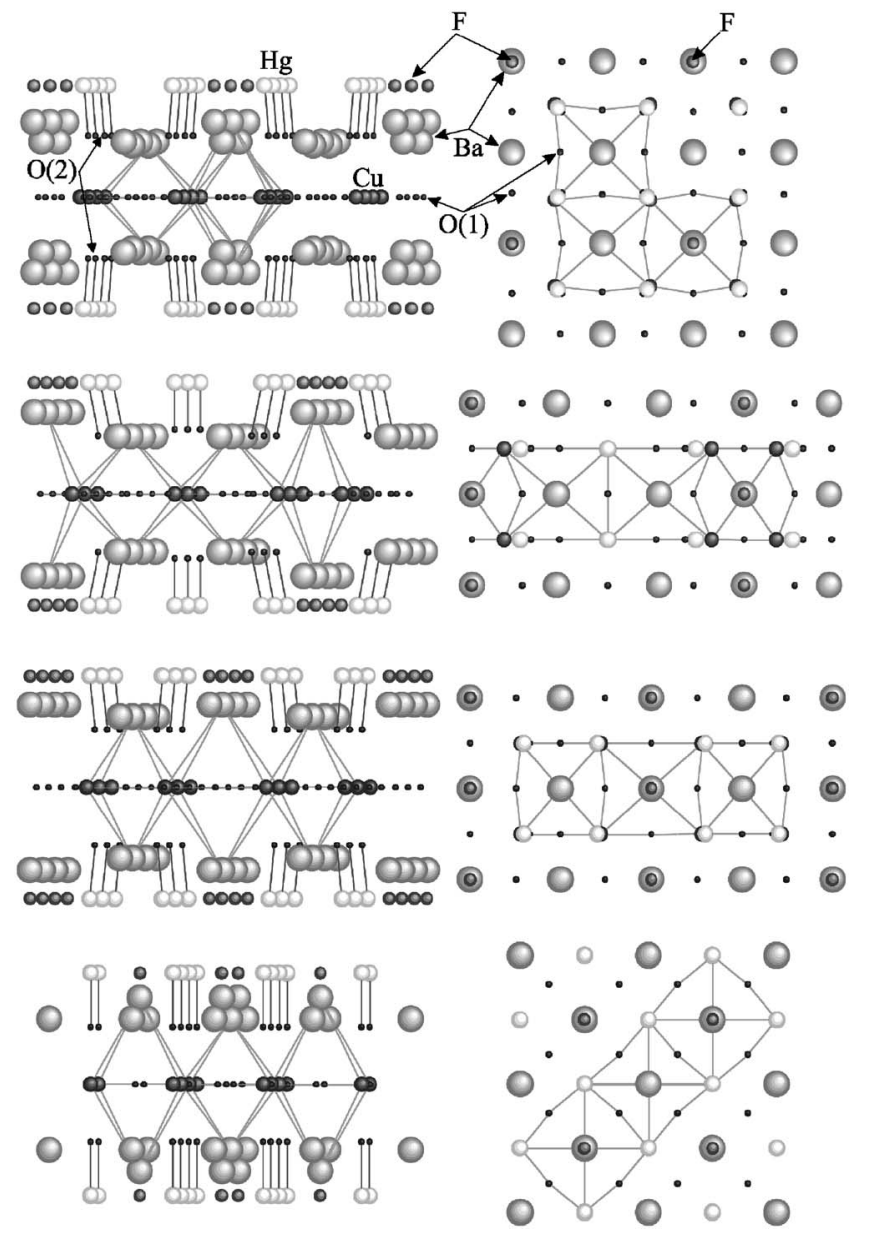

FIG. 4. Artistic view of the Hg1201 supercells structures $D 4, D 3, D 2 b$, and $D 2 a$, as obtained from the FLAPW simulations, which were used to calculate the EFGs. To visually enhance the deformations, the atomic shifts are plotted AMPLIFIED by a factor of 10, while $a$ and $c$ are kept constant. On the right side are shown projections of the lattice along the $c$ axis to emphasize the fluorine atomic positions in the $\mathrm{Hg}$ planes. Resulting deformations on the $\mathrm{CuO}(1)$ planes are stressed by straight lines connecting $\mathrm{Cu}$ to $\mathrm{O}(1)$ atoms. The figures on the left are projections of the lattice along a plane perpendicular to the $c$ axis. In this view the huge shifts of the $\mathrm{Ba}$ atoms towards the negative fluorine ion are clearly seen. Lines connecting $\mathrm{Ba}$ to $\mathrm{Cu}$ atoms are shown, which further stress these deformations. Additional lines connect the apical $\mathrm{O}(2)$ oxygen atoms to the $\mathrm{Hg}$ atoms, revealing the tilts of the $\mathrm{O}(2)-\mathrm{Hg}-\mathrm{O}(2)$ dumbbell due to the neighboring dopant fluorine atoms.

and 1c show a very similar PAC pattern, as do samples 1d and 1e. In all cases the quantitative analysis has proven that in the spectra one single component is dominant. These have been called $\mathrm{EFG}_{1}$ for sample $1(\mathrm{a}), \mathrm{EFG}_{3}$ for samples $1(\mathrm{~b}, \mathrm{c})$ and $\mathrm{EFG}_{4}$ for samples $1(\mathrm{~d}, \mathrm{e})$. It is then highly significant that $\mathrm{EFG}_{1}^{\prime} \equiv \mathrm{EFG}_{1}$ is present as a minor component in samples $1(\mathrm{~d}, \mathrm{e})$ and $\mathrm{EFG}_{4}$ in samples $1(\mathrm{~b}, \mathrm{c})$. This observation will help us to assign the different components to well defined local configurations. We can then use the quantitative component content from the analysis of the PAC spectra to find the doping level of all samples in the probing regions.

For the essentially pure $\mathrm{Hg} 1201$, sample 1(a), one expects the highest EFG with $\eta$ equal to zero for symmetry reasons,

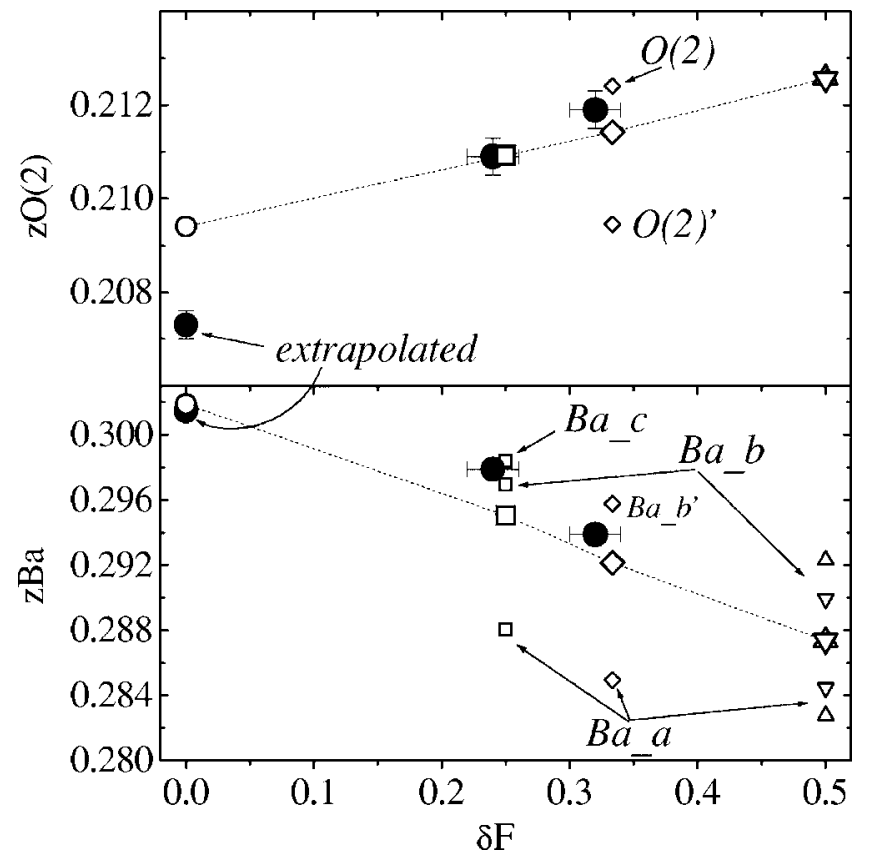

FIG. 5. $z \mathrm{O}(2)$ (top) and $z \mathrm{Ba}$ (bottom) relative coordinates plotted as a function of $\delta \mathrm{F}$. Solid symbols are experimental data from neutron refinement in Ref. 11. Solid symbols at $\delta=0$ are extrapolated from data in Ref. 15. Open symbols describe the parameters obtained from FLAPW relaxation simulations, plotted as a function of the superlattices nominal $\delta \mathrm{F}$. Several displacement values exist for a certain $d F$, which correspond to nonequivalent atoms occurring with different multiplicities. Their weighted average is also shown as bigger open symbols, connected by an eye guide line. It is interesting to observe that the averages approach quite well the experimental data, probably due to the fact that diffraction techniques do not resolve such nonequivalent sites.

as observed in Table II. The parameters are in perfect agreement with our earlier work. The highest doping level ever achieved for fluorine doping is below $50 \%$. We can therefore safely assume that samples $1(\mathrm{~d}, \mathrm{e})$ have a considerably lower value after the fluorine loss during annealing under argon. Since the unperturbed environment is observed only as a minor component, a large fraction of $\mathrm{Hg}$ with one $\mathrm{F}$ neighbor can be assumed. The component $\mathrm{EFG}_{4}$ would be expected to be representative for such an environment, since our theoretical results have demonstrated that distant fluorine dopants lead to a negligible change of the EFG. From the FLAPW calculations an EFG with reduced $\left|V_{z z}\right|$ and $\eta$ approximately 0.2 can be expected. This is exactly the result for $\mathrm{EFG}_{4}$. We can thus safely assign $\mathrm{EFG}_{4}$ to a configuration of one $\mathrm{F}$ neighbor to $\mathrm{Hg}$. With a fraction $f_{4}=70 \%$ and $87 \%$ in the samples $1(\mathrm{~d}, \mathrm{e})$ showing this frequency, the other component being the pure $\mathrm{Hg} 1201$ frequency and a minor unassigned contribution, the PAC data yield a $\delta \mathrm{F} \sim 0.18$ and 0.22 in the probing zone.

Obviously the fraction with $\mathrm{EFG}_{3}$ in samples $1(\mathrm{~b}, \mathrm{c})$ is then representative of more than one $\mathrm{F}$ atom in the $\mathrm{Hg}$ neighborhood. Since the typical doping level achieved for $\mathrm{Hg} 1201$ with fluorination by $\mathrm{XeF}_{2}$ is below $50 \%$, it is straightforward to assume that the fraction showing $\mathrm{EFG}_{3}$ is representative of two $\mathrm{F}$ atoms next to $\mathrm{Hg}$. The reduced value of $\mathrm{EFG}_{3}$ relative 
to $\mathrm{EFG}_{4}$ is consistent with the expectation from our theoretical calculations. The observed $\eta$ of approximately zero is then in favor of these being in adjacent sites. Excellent agreement of the $\mathrm{EFG}_{3}$ parameters with the ones calculated for the mercury sites in the $D 2 b$ configuration is thus observed. The interpretation that the experimentally found $\mathrm{EFG}_{3}$ corresponds to mercury nuclei interacting with two adjacent fluorine atoms positioned at the center of the mercury mesh and aligned in rows parallel to the [100], [010] directions is then the only logical conclusion. It is clear that a configuration similar to $D 2 a$ with fluorine distributed in a chessboardlike pattern is experimentally excluded, since such a high asymmetry parameter, $\eta(\mathrm{Hg})_{D 2 a}=0.416$, has never been observed either for fluorine or for oxygen doped samples. The measured fractions $f_{3}=68 \%, 80 \%$ and $f_{4}$ $=32 \%, 20 \%$ allow us to estimate $\delta \mathrm{F} \sim 0.42=0.34+0.08$ and $\delta \mathrm{F} \sim 0.45=0.40+0.05$ in the probing zone of these experiments. Being slightly higher than the highest doping reported for $\delta \mathrm{F}$ in $\mathrm{Hg} 1201$ by other experiments, this value might be due to our relative higher fluorine concentration in the sealed copper tube during experiments. Still the amount of ionic charge is about the same as reported for the maximum oxygen doping levels in $\mathrm{Hg} 1201$ with $\delta \mathrm{O} \sim 0.22$.

\section{CONCLUSIONS}

The present set of PAC measurements shows clearly that fluorine dopants in $\mathrm{Hg} 1201$ occupy interstitial sites in the $\mathrm{Hg}$ plane at all concentrations. Comparison of the data at the highest doping level with FLAPW calculations demonstrates that the fluorine atoms tend to order along interstitial rows parallel to the [100], [010] directions in the mercury planes, already at room temperature. This must be a short-range effect, however, and the order cannot build up coherently too far and thus originate in a strongly deformed superlattice that would be clearly seen by diffraction techniques. Based on the present charge density simulations the distribution of charge in the copper planes appears to be rather uncoupled from the dopant ordering, possibly due to the large number of closely spaced d-states at the copper atoms. Therefore this work further supports the model that the origin of charge and magnetic stripes must be an essential phenomenon of the strong antiferromagnetic copper-copper coupling, with small influence from pure atomistic deformations of the lattice structure. The present work is being extended to $\operatorname{Hg} 1212(n=2)$ and $\operatorname{Hg} 1223(n=3)$ where high dopant oxygen concentrations are achievable, with measurements foreseen in a large temperature range covering the superconducting transition.

\section{ACKNOWLEDGMENTS}

We acknowledge the support of FCT, Portugal (Project No. PDCT-FP-FNU-50145-2003). E.R. and A.M.L.L. acknowledge the support of FCT.
*On leave from Phys. Dept. Instituto Tecnológico e Nuclear, E.N. 10, 2686-953, Sacavém, Portugal.

${ }^{\dagger}$ Author to whom correspondence should be addressed. Electronic address: guilherme.correia@cern.ch

${ }^{1}$ X. G. Zheng, C. N. Xu, Y. Tomokiyo, E. Tanaka, H. Yamada, and Y. Soejima, Phys. Rev. Lett. 85, 5170 (2000).

${ }^{2}$ H. A. Mook, P. Dai, and F. Dogan, Phys. Rev. Lett. 88, 097004 (2002); Y. Ando, K. Segawa, S. Komiya, and A. N. Lavrov, ibid. 88, 137005 (2002).

${ }^{3}$ S. A. Kivelson, I. P. Bindloss, E. Fradkin, V. Oganesyan, J. M. Tranquada, A. Kapitulnik, and C. Howald, Rev. Mod. Phys. 75, 1201 (2003); more refs. in http://focus.aps.org/story/v9/st12.

${ }^{4}$ J. M. Tranquada, H. Wool, T. G. Perring, H. Goka, G. D. Gul, G. Xul, M. Fujita, and K. Yamada, Nature (London) 429, 534 (2004), and references therein.

${ }^{5}$ S. N. Putilin, E. V. Antipov, O. Chmaissem, and M. Marezio, Nature (London) 362, 226 (1993); A. Schilling, M. Cantoni, J. D. Guo, and H. R. Ott, ibid. 363, 56 (1993).

${ }^{6}$ M.-H. Julien, P. Carretta, M. Horvatic, C. Berthier, Y. Berthier, P. Ségransan, A. Carrington, and D. Colson, Phys. Rev. Lett. 76, 4238 (1996); M.-H. Julien, M. Horvatić, P. Carretta, C. Berthier, Y. Berthier, P. Ségransan, S. M. Loureiro, and J.-J. Capponi, Physica C 268, 197 (1996); C. H. Booth, F. Bridges, E. D. Bauer, G. G. Li, J. B. Boyce, T. Claeson, C. W. Chu, and Q. Xiong, Phys. Rev. B 52, R15745 (1995).

${ }^{7}$ J. T. Market, Y. Dalichaouch, and M. B. Maple, in Physical Properties of High Temperature Superconductors I, edited by D. M. Ginsberg (World Scientific, Singapore, 1989), p. 265; C. L.
Chien, G. Xiao, M. Z. Cieplak, D. Musser, J. J. Rhyne, and J. A. Gotaas, in Superconductivity and Its Applications, edited by $\mathrm{H}$. S. Kwok, and D. T. Shaw (Elsevier, New York, 1988), p. 110.

${ }^{8}$ S. M. Loureiro, J. J. Capponi, E. V. Antipov, and M. Marezio, Studies of High Temperature Superconductors, edited by A. V. Narlikar (Nova Science, New York, 1997), Vol. 25 and references therein.

${ }^{9}$ A. Lanzara, N. L. Saini, A. Bianconi, F. Duc, and P. Bordet, Phys. Rev. B 59, 3851 (1999).

${ }^{10}$ P. Bordet, F. Duc, P. G. Radaelli, A. Lanzara, N. Saini, A. Bianconi, and E. V. Antipov, Physica C 282-287, 1081 (1997).

${ }^{11}$ A. M. Abakumov, V. L. Aksenov, V. A. Alyoshin, E. V. Antipov, A. M. Balagurov, D. A. Mikhailova, S. N. Putilin, and M. G. Rozova, Phys. Rev. Lett. 80, 385 (1998); S. N. Putilin, E. V. Antipov, A. M. Abakumov, M. G. Rozova, K. A. Lokshin, D. A. Pavlov, A. M. Balagurov, D. V. Sheptyakov, and M. Marezio, Physica C 338, 52 (2000).

${ }^{12}$ C. W. Chu, L. Gao, F. Chen, Z. J. Huang, R. L. Meng, and Y. Y. Xue, Nature (London) 365, 323 (1993); M. Nunez-Regueiro, J. L. Tholence, E. V. Antipov, J. J. Capponi, and M. Marezio, Science 262, 97 (1993).

${ }^{13}$ J. G. Correia, the ISOLDE collaboration J. P. Araújo, S. M. Loureiro, P. Toulemonde, S. Le Floch, P. Bordet, J. J. Capponi, R. Gatt, W. Tröger, B. Ctortecka, T. Butz, H. Haas, J. G. Marques, and J. C. Soares, Phys. Rev. B 61, 11769 (2000).

${ }^{14}$ M. Coey, Nature (London) 430, 155 (2004).

${ }^{15}$ V. A. Alyoshin, D. A. Mikhailova, and E. V. Antipov, Physica C 271, 197 (1996). 
${ }^{16}$ E. Kugler, D. Fiander, B. Jonson, H. Haas, A. Przewloka, H. L. Ravn, D. J. Simon, K. Zimmer, and the ISOLDE collaboration, Nucl. Instrum. Methods Phys. Res. B 70, 41 (1992).

${ }^{17}$ J. F. Ziegler, J. P. Biersack, and U. Littmark, The Stopping and Range of Ions in Solids (Pergamon, New York, 1985).

${ }^{18}$ T. Butz, S. Saibene, Th. Fraenzke, and M. Weber, Nucl. Instrum. Methods Phys. Res. A 284, 417 (1989).

${ }^{19}$ N. P. Barradas, M. Rots, A. A. Melo, and J. C. Soares, Phys. Rev. B 47, 8763 (1993).

${ }^{20}$ M. Deicher, Hyperfine Interact. 79 (1993) 681.
${ }^{21}$ P. Blaha, P. Dufek, K. Schwarz, and H. Haas, Hyperfine Interact. 97/98, 3 (1996); P. Blaha, K. Schwarz, and J. Luitz, wIEN97, Vienna Univ. of Tech. 1997 in P. Blaha, K. Schwarz, and P. SorantinS. B. Trickey, Comput. Phys. Commun. 59, 399 (1990).

${ }^{22}$ J. P. Perdew, K. Burke, and M. Ernzerhof, Phys. Rev. Lett. 77, 3865 (1996).

${ }^{23}$ A. M. Balagurov, D. V. Sheptyakov, V. L. Aksenov, E. V. Antipov, S. N. Putilin, P. G. Radaelli, and M. Marezio, Phys. Rev. B 59, 7209 (1999).

${ }^{24}$ D. J. Singh and W. E. Pickett, Phys. Rev. Lett. 73, 476 (1994). 